\title{
Angewandte Geographie in der Schweiz - Versuch einer Standortbestimmung
}

Noch vor wenigen Jahren sind angewandt tätige Geographen beinahe als exotische Exemplare einer Berufsgattung betrachtet worden, welche sich gefälligst auf den ihr zugewiesenen Platz im Schuldienst zu beschränken habe. Mit der zunehmenden Gestaltung und Veränderung unserer Landschaft - vor allem mit den vermehrt als negativ erkannten Folgen auf unseren Lebensraum und seine Naturgrundlagen hat sich die Überzeugung durchgesetzt, im Rahmen der angewandten Geographie forschend, planend und ordnend beim Schutz der natürlichen Lebensgrundlagen und an weiteren räumlichen Umgestaltungen zu einer optimalen Umwelt für die Menschheit beitragen zu können. Damit gewinnt die angewandte Geographie - worunter wir hier alle Tätigkeiten der Geographie außerhalb der Aus- und Weiterbildung verstehen wollen - vor allem in den aktuellen Bereichen der Raumplanung und des Umweltschutzes zunehmend an Bedeutung. Wir können heute in der Politik und in den Medien fast täglich feststellen, welch hoher Stellenwert Problemen der angewandten Geographie in unserem Land zukommt.

Es erstaunt deshalb auch nicht, daß gerade unser Fach in den letzten Jahren an den schweizerischen Hochschulen eine enorme Nachfrage zu verzeichnen hatte (mehr als Vervierfachung der Studienanfänger zwischen 1968 und 1979). Eine Umfrage, welche die Schweizerische Gesellschaft für angewandte Geographie 1982 bei allen Geographischen Instituten unseres Landes durchgeführt hat, zeigt mit aller Deutlichkeit, daß wir in allernächster Zukunft bei den Geographieabsolventen, welche später eine Tätigkeit außerhalb der Schule ausüben wollen, mit einem Mehrfachen dessen rechnen müssen, was an entsprechenden Stellen überhaupt zur Verfügung stehen wird. Aufgrund des verfügbaren Zahlenmaterials läßt sich abschätzen, daß die Zahl der Absolventen, welche 1982/83 abgeschlossen haben, bereits rund einem Drittel aller durch angewandt tätige Geographen insgesamt besetzten Stellen gleichkommt. Nicht vergessen werden darf bei der Beurteilung dieser Tatsache, daß die natürliche Stellenrotation noch kaum spielt, weil das Durchschnittsalter der heutigen Stelleninhaber relativ niedrig ist.

Mit der zunehmenden Verschärfung dieser Situation stehen wir in der Schweiz nicht isoliert da. Ein Blick über die Grenzen zeigt uns, daß man beispielsweise in der Bundesrepublik Deutschland die jährliche $\mathrm{Zu}$ - nahme an Geographieabsolventen, welche später angewandt tätig sein wollen, auf $20 \%$ schätzt. Im Studienjahr 1982/83 wurden in diesem Bereich rund 7000 Studierende gezählt; eine Nachfrage an Arbeitsmöglichkeiten, welcher bei sehr optimistischer Beurteilung bestenfalls einige hundert Stellen entgegenstehen.

Angesichts dieser Situation - einerseits die Aktualität von Problemen im Bereich der angewandten Geographie, anderseits die rapid sinkende Wahrscheinlichkeit, nach dem Geographiestudium eine adäquate Stelle zu finden - ist die Frage berechtigt: Findet Geographie zunehmend ohne Geographen statt? Wenn man feststellt, daß Bundesämter, welche maßgeblich in den hier angesprochenen Bereichen tätig sind, keinen einzigen Geographen beschäftigen, und da $ß$ in Forschungsgremien für uns zentrale Anliegen nicht von Geographen vertreten werden, ist man geneigt, diese Frage zu bejahen.

Auf der andern Seite darf nicht übersehen werden, daß angewandte Geographen in sehr vielen Bereichen unseres Faches tätig sind und mit ihrer Arbeit das Berufsbild des Geographen verbreiten helfen. Gemäß einer 1981 unter den Mitgliedern der Schweizerischen Gesellschaft für angewandte Geographie durchgeführten Umfrage arbeiten die Befragten bei folgenden Stellen:

\begin{tabular}{lrc|} 
Verwaltung & & $46 \%$ \\
- Bundesebene & $20 \%$ & \\
- Kantonsebene & $20 \%$ & \\
- Regionsebene & $4 \%$ & \\
- städtische Ebene & $2 \%$ & \\
Hochschule & & $24 \%$ \\
Privatwirtschaft & & $22 \%$ \\
- Planungs- und Beratungsbüros & $11 \%$ & \\
- andere Dienstleistungsbetriebe & $4 \%$ & \\
- selbständige Tätigkeit & $7 \%$ & \\
Andere & & $8 \%$ \\
\hline
\end{tabular}

Ulrich Roth, Präsident der Schweizerischen Gesellschaft für angewandte Geographie

Sigmaplan, Zähringerstraße 61, 3012 Bern 
Auf Tätigkeitsgebiete aufgeteilt ergibt sich ungefähr folgendes Bild:

\begin{tabular}{lr|} 
Raumplanung (inkl. Sachplanungen) & $39 \%$ \\
Regionalwirtschaft & $7 \%$ \\
Natur- und Landschaftsschutz & $17 \%$ \\
übriger Umweltschutz & $1 \%$ \\
Grundlagen und angewandte Forschung & $16 \%$ \\
Dokumentation & $3 \%$ \\
Kartographie, Verlagswesen & $4 \%$ \\
Museumswesen & $3 \%$ \\
Journalismus & $1 \%$ \\
Übrige & $9 \%$ \\
\hline
\end{tabular}

Wir dürfen also feststellen, daß bereits in einem breiten Spektrum von Tätigkeiten Geographen Eingang gefunden haben und vielerorts als ernstzunehmende Partner akzeptiert sind. So wurde beispielsweise die Schweizerische Gesellschaft für angewandte Geographie von den Bundesämtern für Landwirtschaft und Raumplanung mit den Vorbereitungen für ein - inzwischen vom Bundesrat genehmigtes Nationales Forschungsprogramm «Nutzung des Bodens» betraut.

Das vorliegende Themenheft über angewandte Geographie in der Schweiz erscheint aus Anlaß eines Symposiums im Zusammenhang mit dem Internationalen Geographenkongress in Paris, welches vom 20. bis 25. August 1984 in Zürich und Lausanne stattfindet und von der Schweizerischen Gesellschaft für angewandte Geographie in Zusammenarbeit mit der Eidgenössischen Technischen Hochschule und der Universität Lausanne organisiert wird. Dahinter steht die Absicht, aus verschiedenen Blickwinkeln Möglichkeiten und Randbedingungen für Geographen zu beleuchten und damit eine Standortbestimmung bezüglich ihrer Integration in unterschiedlichen Tätigkeitsbereichen vorzunehmen.

Im Sinne einer Absichtserklärung sollen die folgenden Forderungen zur Diskussion gestellt werden:

- Engere Zusammenarbeit zwischen Hochschule und Praxis, damit die effektiven Bedürfnisse vermehrt und rechtzeitig in die Ausbildung einfließen können. Dies kann beispielsweise mittels vermehrter Praktika außerhalb der Hochschule im Rahmen des Studiums einerseits und mit Lehraufträgen an Praktiker anderseits erfolgen.

- Verbesserte Informationen der Studenten über Arbeitsmöglichkeiten und deren spezifische Anforderungen; zum Beispiel durch eine ständige Aktualisierung des Lehrstoffes und der Institutsbibliotheken mit Unterlagen aus der Praxis, wodurch auch persönliche Kontakte zu den entsprechenden Bearbeitern erleichtert werden könnten.

- Spezialisierung in einem Teilgebiet der Geographie anstreben, ohne aber die Zusammenhänge mit andern Fachbereichen zu vergessen. Es stimmt zwar, daß sich Generalisten im Rahmen von Projektmanagements Möglichkeiten öffnen können, der Einstieg ins Berufsleben beginnt aber praktisch immer auf Sachbearbeiterebene; und hier sind vor allem Spezialisten gefragt, welche dem großen Konkurrenzdruck von Nachbardisziplinen standhalten können.

- Vermehrte Imagepflege und Zusammenarbeit aller angewandt tätigen Geographen. Warum bekennen sich zum Beispiel - laut Umfrage der Schweizerischen Gesellschaft für angewandte Geographie mehr als die Hälfte unserer Berufskollegen in ihrer Berufsbezeichnung nicht zur Geographie? Es tut not, daß wir unsere Arbeiten und Tätigkeiten unter diesem Aspekt vermehrt einem breiten Publikum präsentieren und damit Aufmerksamkeit lenken auf die Möglichkeiten der Geographen zur Lösung anstehender Probleme.

Mit dem vorliegenden Heft beabsichtigen die Autoren, zum einen unter den Geographen selbst, zum andern aber auch in einer breiteren Öffentlichkeit eine Diskussion anzuregen. Ein derartiger Meinungsaustausch scheint uns notwendig, wollen wir die genannten Forderungen nicht weiterhin lediglich als Lippenbekenntnisse weiterverbreiten. Wir dürfen uns daher glücklich schätzen, für diese Aufgabe kompetente Autoren aus unseren eigenen Reihen und uns nahestehenden Kreisen gewonnen zu haben. Selbstverständlich würde es den Rahmen nicht nur dieser Publikation sprengen, alle Aspekte der angewandten Geographie in der Schweiz beleuchten zu wollen. Im Interesse der Übersichtlichkeit haben wir die 15 Artikel in sechs Gruppen gegliedert:

1. Angewandte Geographie - Stellenwert in der Raumplanung

2. Angewandte Geographie - Zusammenarbeit zwischen Forschung und Praxis

3. Angewandte Geographie - Grundlagen zu aktuellen Problemen

4. Angewandte Geographie im Rahmen der Beurteilung von Großprojekten

5. Angewandte Geographie - Beitrag zum Natur- und Landschaftsschutz

6. Angewandte Geographie in der öffentlichen Auseinandersetzung

Leider ist es uns nicht gelungen, welsche Kollegen für eine Mitarbeit zu animieren, obwohl die angewandte Geographie auch im französisch- und italienischsprachigen Teil der Schweiz wertvolle Beiträge zur Lösung aktueller Probleme leistet.

Wir hoffen, mit dem vorliegenden Heft auf die vielseitigen Tätigkeitsgebiete der angewandten Geographie in der Schweiz aufmerksam zu machen. Vor allem aber liegt uns daran zu zeigen, daß den Geographen noch Möglichkeiten erschlossen werden könnten, falls alle interessierten Kreise mit der erforderlichen Entschlossenheit und dem uneigennützigen 
Willen zur Zusammenarbeit das gleiche Ziel verfolgen. Dies wird eine der vordringlichsten Aufgaben der angewandten Geographie für die kommenden Jahre sein.

Zum Schluß bleibt uns die angenehme Pflicht, allen am Zustandekommen dieses Heftes Beteiligten herz- lich zu danken. In erster Linie sind dies die Autoren, die sich spontan bereit erklärt haben, ihre wertvolle Erfahrung hier einfließen zu lassen. Danken möchten wir aber auch der Redaktion der Geographica Helvetica, welche uns bereitwillig die vorliegende Publikation ermöglicht hat.

\title{
La géographie appliquée en Suisse - réflexions sur sa position dans le champ des activités de notre société
}

\author{
Traduction: Cornelia Mayerhofer, Zurich
}

Il n'y a pas longtemps que les géographes actifs en geographie appliquée étaient presque regardés comme les exemplaires exotiques d'un groupe professionnel qui n'aurait d'autres places que dans l'enseignement. Le développement et la transformation toujours plus intensifs de notre paysage - et surtout leurs conséquences qui s'avèrent de plus en plus négatives pour notre environnement et les bases naturelles de la vie ont entre-temps convaincu le public que la géographie appliquée a le pouvoir de contribuer à la protection des bases naturelles de la vie et à la création d'un environnement optimal pour l'homme par l'aménagement continu et par la recherche, le planning et l'organisation. La géographie appliquée - et par là nous entendons toutes les activités géographiques en dehors de l'enseignement et la formation permanente - joue donc un rôle toujours plus important dans notre société, avant tout dans les domaines actuels de l'aménagement du territoire et de la protection de l'environnement. La politique et les medias nous montrent presque tous les jours l'actualité des problèmes dans le domaine de la géographie appliquée de notre pays.

A cet égard, il n'est pas surprenant que le nombre des étudiants en géographie inscrits aux universités et écoles polytechniques suisses s'est plus que quadruplé entre 1968 et 1979. Un sondage de la Société suisse de géographie appliquée, qui en 1982 questionna tous les instituts géographiques de notre pays, a clairement révélé que le nombre de nouveaux diplômés en géographie qui visent à gagner leur vie en dehors de l'enseignement dépassera bientôt largement le nombre des emplois offerts. Selon ces données l'on peut estimer que le nombre des étudiants diplômés en 1982/83 égale déjà un tiers de tous les emplois occupés par des géographes. S'y ajoute le fait que la rotation naturelle des emplois ne joue pas encore un rôle à cause de la moyenne d'âge relativement basse des employés.

La Suisse n'est cependant pas le seul pays où la situation s'aggrave. En Allemagne fédérale, par exemple, l'on estime que le nombre de nouveaux diplômés qui visent à gagner leur vie en dehors de l'enseignement augmente de $20 \%$ par an. En 1982/83, cette discipline comptait environ 7000 étudiants, une demande en emplois à laquelle ne s'opposent, dans le cas le plus favorable, que quelques centaines de places.

Vu cette situation - l'actualité des problèmes dans le domaine de la géographie appliquée d'une part et l'impossibilité croissante de trouver un emploi adéquat d'autre part, l'on peut bien se demander si la géographie va de plus en plus avoir lieu sans des géographes. Si l'on constate que les offices fédéraux, qui s'occupent dans une large mesure des domaines de la géographie appliquée, n'emploient aucun géographe et que, dans les organes de recherches, des tâches centrales de notre discipline ne sont pas traitées par des géographes, l'on est incliné à affirmer cette question.

Il ne faut par contre pas oublier que des géographes sont actifs dans de nombreux domaines de notre discipline et aident ainsi à répandre l'image professionnelle des géographes par leur travail. Selon un sondage fait parmi les membres de la Société suisse de géographie appliquée en 1981, les géographes questionnés occupent les positions suivantes:

\begin{tabular}{|lrr|}
\hline Administration & & $46 \%$ \\
- au niveau fédéral & $20 \%$ & \\
- au niveau cantonal & $20 \%$ & \\
- au niveau régional & $4 \%$ & \\
- au niveau municipal & $2 \%$ & \\
Ecoles polytechniques/universités & & $24 \%$ \\
Industrie privée & & $22 \%$ \\
- bureaux d'aménagement et de & & \\
$\quad$ consultation & $11 \%$ & \\
- autres entreprises du secteur tertiaire & $4 \%$ & \\
- géographes indépendants & $7 \%$ & \\
Autres & & $8 \%$ \\
\hline
\end{tabular}

par Ulrich Roth, président de la Société Suisse de Géographie appliquée 